\title{
A new method to obtain decay rate estimates for dissipative systems with localized damping.
}

\author{
Patrick MARTINEZ
}

\begin{abstract}
We consider the wave equation damped with a locally distributed nonlinear dissipation. We improve several earlier results of $E$. Zuazua and of $M$. Nakao in two directions: first, using the piecewise multiplier method introduced by $\mathrm{K}$. Liu, we weaken the usual geometrical conditions on the localization of the damping. Then thanks to some new nonlinear integral inequalities, we eliminate the usual assumption on the polynomial growth of the feedback in zero and we show that the energy of the system decays to zero with a precise decay rate estimate.
\end{abstract}

\section{Introduction}

The problem of stabilization of the wave equation in a bounded domain with the Dirichlet boundary condition by the use of a locally distributed damping has been studied by several authors. Consider the following system

$$
\left\{\begin{array}{l}
u^{\prime \prime}-\Delta u+\rho\left(x, u^{\prime}\right)=0 \text { in } \Omega \times \boldsymbol{R}_{+}, \\
u=0 \text { on } \partial \Omega \times \boldsymbol{R}_{+}, \\
u(0)=u^{0}, u^{\prime}(0)=u^{1} .
\end{array}\right.
$$

Define the energy of the system by

$$
E(t)=\frac{1}{2} \int_{\Omega}\left(u^{\prime 2}+|\nabla u|^{2}\right) d x .
$$

1991 Mathematics Subject Classification: 26A12, 35B40, 93D15.

Servicio Publicaciones Univ. Complutense. Madrid, 1999. 
C.M. Dafermos [5] and A. Haraux [10] studied the strong stability of this system. M. Aassila [1] extended some of their results in the case of unbounded domains.

When the feedback term depends on the velocity in a linear way, i.e.

$$
\rho\left(x, u^{\prime}\right)=a(x) u^{\prime},
$$

E. Zuazua [23] proved that the energy decays exponentially if the damping region, where $a(x) \geq \alpha>0$, contains a neighbourhood of $\partial \Omega$ or at least a neighbourhood of

$$
\Gamma\left(x^{0}\right):=\left\{x \in \partial \Omega,\left(x-x^{0}\right) \cdot \nu(x) \geq 0\right\},
$$

where $\nu$ is the outward unit normal to $\Omega$ and $x^{0} \in R^{N}$. His proof is based on the multiplier method and on a compactness argument. On the other hand, C. Bardos, G. Lebeau and J. Rauch [3] obtained a necessary and sufficient condition using microlocal analysis: the energy decays exponentially if and only if the damping region satisfies the "geometric optics condition".

M. Nakao extended the results of E. Zuazua [23] treating first the case of a linear degenerate dissipation ([18]), and then the case of a nonlinear dissipation ([19]), assuming as usually that the function $\rho(x, \cdot)$ has a polynomial growth in zero.

M. Aassila [2] extended some results of M. Nakao [17] concerning weak dissipations.

L.R. Tcheugoué Tébou [21] improved slightly the estimates of M. Nakao [19].

Recently K. Liu [14] studied the observability of a class of P.D.E.s in a internal subdomain. He extended the results of E. Zuazua [22] weakening the geometrical conditions on the observation region: using a piecewise multiplier method he obtained very general and easily verifiable geometrical conditions. K. Liu and M. Yamamoto [15] adapted this method and norm inequalities developped by V. Komornik [11] to establish the observability of the system of the wave equation with the Dirichlet boundary condition under the same geometrical conditions with very precise estimates on the time duration $T$.

In the following we introduce a new method useful to eliminate the assumption of the polynomial growth of $\rho(x, \cdot)$. We combine this method 
to the piecewise multiplier techniques developped by K. Liu [14] to show that the energy of the system decays to zero with a precise decay rate estimate if the damping region satisfies some geometrical conditions. This method can also be used to study the case of the wave equation damped with a velocity feedback on the boundary (see [16]). Under some geometrical conditions, and without assuming that the feedback has a polynomial growth in zero, this method gives a more explicit decay rate estimate than the result of I. Lasiecka and D. Tataru [12], who studied the more general case of a semilinear wave equation damped with a nonlinear velocity feedback acting on a part of the boundary. Without any geometrical condition and without assuming that the feedback has a polynomial growth in zero, they showed that the energy decays as fast as the solution of some associated differential equation.

In Section 2, we precise our results (see Theorem 1) and we apply it on some examples. In Section 3, we give an analogous result for elastodynamic systems. In Section 4, we establish the new integral inequalities useful to prove our result. Section 5 contains the technical part of the proof; its goal is to prove Proposition 5.1. In Section 6 we conclude using Proposition 5.1 and Lemma 3.

\section{Statement of the problem and main results}

Let $\Omega$ be a bounded open set of $\boldsymbol{R}^{N}$ of class $\mathcal{C}^{2}$ and $\omega$ be a subset of $\Omega$. Let $\rho: \bar{\Omega} \times \boldsymbol{R} \longrightarrow \boldsymbol{R}$ be a continuous function. Denote $\boldsymbol{R}_{+}:=[0,+\infty[$. Consider the following evolutionary problem

$$
\begin{gathered}
u^{\prime \prime}-\Delta u+\rho\left(x, u^{\prime}\right)=0 \text { in } \Omega \times \boldsymbol{R}_{+}, \\
u=0 \text { on } \partial \Omega \times \boldsymbol{R}_{+} \\
u(0)=u^{0}, u^{\prime}(0)=u^{1} .
\end{gathered}
$$

As usually, we define the energy of the solution $u$ by:

$$
E(t)=\frac{1}{2} \int_{\Omega}\left(u^{\prime 2}+|\nabla u|^{2}\right) d x .
$$

We will use the following notations:

- $x \cdot y$ represents the euclidean scalar product of $x$ by $y$ in $\boldsymbol{R}^{N}$, 
- if $\Omega_{j} \subset \boldsymbol{R}^{N}$ is a Lipschitz domain, $\nu_{j}$ represents the outward unit normal vector to $\partial \Omega_{j}$,

- if $\mathcal{O} \subset \mathbb{R}^{N}$ and $x \in \mathbb{R}^{N}, d(x, \mathcal{O})=\inf _{y \in \mathcal{O}}|x-y|$, and $\mathcal{N}_{\varepsilon}(\mathcal{O}):=$ $\left\{x \in \boldsymbol{R}^{N}: d(x, \mathcal{O})<\varepsilon\right\}$.

We make the following assumptions:

Hyp. 1: assume that $\rho:(x, v) \mapsto \rho(x, v)$ belongs to $\mathcal{C}(\bar{\Omega} \times \mathbb{R})$ and is monotone increasing in $v$. Assume that there exist a nonnegative bounded function $a: \Omega \longrightarrow R$ and a strictly increasing odd function $g: \boldsymbol{R} \longrightarrow \boldsymbol{R}$ of class $\mathcal{C}^{1}$ such that

$$
\begin{gathered}
a(x)|v| \leq|\rho(x, v)| \leq C a(x)|v| \text { if }|v| \geq 1, \\
a(x) g(|v|) \leq|\rho(x, v)| \leq C a(x) g^{-1}(|v|) \text { if }|v| \leq 1,
\end{gathered}
$$

where $g^{-1}$ denotes the inverse function of $g$ and $C$ is a positive constant. Denote

$$
G(v)=v g(v) \text { and } H(v)=\frac{g(v)}{v} .
$$

Note that $G$ is continuous, strictly increasing on $[0,1]$ and $G(0)=0 ; H$ is continuous on $[0,1]$ and $H(0)=g^{\prime}(0)$. M. Nakao [19] treated the case when $g(v)=v^{p}$ for $p>1$.

Hyp. 2: assume the following geometrical conditions on $\Omega$ and $\omega$ as in Liu [14]: there exist $\varepsilon>0$, domains $\Omega_{j} \subset \Omega, 1 \leq j \leq J$ with Lipschitz boundary $\partial \Omega_{j}$ and points $x_{j} \in \boldsymbol{R}^{n}$ such that

$$
\begin{gathered}
\Omega_{i} \cap \Omega_{j}=\emptyset \text { if } i \neq j, \\
\omega \supset \Omega \cap \mathcal{N}_{e}\left[\cup_{j} \Gamma_{j}\left(x_{j}\right) \cup\left(\Omega \backslash \cup_{j} \Omega_{j}\right)\right], \\
\text { where } \Gamma_{j}\left(x_{j}\right):=\left\{x \in \partial \Omega_{j}:\left(x-x_{j}\right) \cdot \nu_{j}(x)>0\right\} .
\end{gathered}
$$

The usual assumption made by E. Zuazua [23] corresponds to the case where we have only one subdomain $\Omega_{1}$ equal to $\Omega$. Denote $m_{j}(x)=$ $x-x_{j}$.

The existence and the regularity of the solution $u$ of $(2.1)-(2.3)$ is given by the following standard proposition (see, e.g. A. Haraux [9]):

Proposition 2.1. Assume that $\Omega$ is of class $\mathcal{C}^{2}$. Under Hypothesis 1 
1. given $\left(u^{0}, u^{1}\right) \in H_{0}^{1}(\Omega) \times L^{2}(\Omega)$, the problem (2.1)-(2.3) admits a unique solution $u(t)$ in the class:

$$
u \in \mathcal{C}\left(\boldsymbol{R}_{+}, H_{0}^{1}(\Omega)\right) \cap \mathcal{C}^{1}\left(\boldsymbol{R}_{+}, L^{2}(\Omega)\right) .
$$

2. given $\left(u^{0}, u^{1}\right) \in H^{2} \cap H_{0}^{1}(\Omega) \times H_{0}^{1}(\Omega)$, the problem (2.1)-(2.3) admits a unique solution $u(t)$ in the class:

$$
u \in W^{2, \infty}\left(\boldsymbol{R}_{+}, L^{2}(\Omega)\right) \cap W^{1, \infty}\left(\boldsymbol{R}_{+}, H_{0}^{1}(\Omega)\right) \cap L^{\infty}\left(\boldsymbol{R}_{+}, H^{2}(\Omega)\right) .
$$

Theorem 1. Assume that $a \in \mathcal{C}^{0}(\bar{\Omega})$ and is bounded from below by some positive constant $\alpha>0$ on $\omega$ :

$$
\forall x \in \omega, \quad a(x) \geq \alpha>0 .
$$

Under Hyp. 1 and Hyp. 2, we have:

1. In the simpler case when $g(v)=v$, the energy decays exponentially: there exists a constant $\gamma=\gamma\left(\Omega, \omega, \alpha, \Omega_{j}, x_{j}\right)>0$ such that, given $\left(u^{0}, u^{1}\right) \in H_{0}^{1}(\Omega) \times L^{2}(\Omega)$, the energy of the solution $u$ of (2.1)-(2.3) satisfies the following estimate:

$$
\forall t \geq 0, E(t) \leq E(0) e^{1-\gamma t} .
$$

2. In the general case, the energy of the solution $u$ of (2.1)-(2.3) decays as

$$
\forall t>0, E(t) \leq C\left(G^{-1}\left(\frac{1}{t}\right)\right)^{2},
$$

with a constant $C$ only depending on the initial energy $E(0)$ (and in a continuous way).

3. Moneover if $H$ is an increasing function and $H(0)=0$, then in fact

$$
\forall t>0, E(t) \leq C\left(g^{-1}\left(\frac{1}{t}\right)\right)^{2}
$$

with a constant $C$ only depending on the initial energy $E(0)$ (and in a continuous way).

Remarks. 1. Part 1 of Theorem 1 improves the geometrical conditions of the result of E. Zuazua [23]. It is not difficult to construct a function $a$ which satisfies (2.13) without satisfying the condition

$$
a(x) \geq \alpha \text { on } \Gamma\left(x^{0}\right),
$$


for example if $\Omega$ is the open ball in $\mathbb{R}^{2}$ centered in $O$ and of radius $R$, and $a$ is a continuous function equal to zero only on the set of points $z \in\{z,|z|=R\} \cap\left\{|\operatorname{Re} z| \leq \frac{R}{3}\right\}$. Define

$$
\begin{gathered}
\Omega_{1}:=\Omega \cap \mathcal{B}\left(2 R i, \frac{3 R}{2}\right) \text { and } x_{1}:=2 R i \\
\Omega_{2}:=\Omega \cap \mathcal{B}\left(-2 R i, \frac{3 R}{2}\right) \text { and } x_{2}:=-2 R i, \\
\omega:=\left\{z \in \Omega:|\operatorname{lm} z| \leq \frac{3 R}{4}\right\} .
\end{gathered}
$$

Then (2.8)-(2.10) are satisfied if $\varepsilon$ is small enough. (see K. Liu [14] for other examples)

The proof is based on the piecewise multiplier method introduced in $\mathrm{K}$. Liu [14] and gives an explicit estimate of the constant $\gamma$.

2. Part 2 and Part 3 of Theorem 1 improve the result of $M$. Nakao [19] in two directions: we weaken the geometrical conditions and we do not assume that $g$ has a polynomial growth in zero. The proof is based on some new integral inequalities stated in Section 4.

For example, let $a$ be a nonnegative continuous function that satisfies (2.13). Then:

Example 1. If

$$
\rho(x, v)=a(x) e^{-1 / v^{p}} \text { if } 0<v<1
$$

for some $p>0$, then (2.16) gives the estimate

$$
E(t) \leq \frac{C}{(\ln t)^{2 / p}}
$$

Example 2. If

$$
\rho(x, v)=a(x) e^{-e^{1 / v}} \text { if } 0<v<1,
$$

then (2.16) gives the estimate

$$
E(t) \leq \frac{C}{(\ln (\ln t))^{2}}
$$




\section{Example 3. If}

$$
\rho(x, v)=a(x) v^{p} \text { if } 0<v<1
$$

for some $p>1$, the method that leads to (2.16) gives by an induction argument:

$$
E(t) \leq C_{\varepsilon} t^{\frac{-2}{p-1}+\varepsilon},
$$

which is close to the estimate found by M. Nakao [19]:

$$
E(t) \leq C t^{\frac{-2}{p-1}} \text {. }
$$

\section{Application to elastodynamic systems}

We can apply the method that lead to prove Theorem 1 to study the following elastodynamic system: let $\left(a_{i j k l}\right)$ be a tensor such that

$$
a_{i j k l}=a_{j i k l}=a_{k l i j}
$$

(all indices run over the integers $1 \ldots \mathrm{N}$ ), satisfying for some $\beta>0$ the ellipticity condition

$$
a_{i j k l} \varepsilon_{i j} \varepsilon_{k l} \geq \beta \varepsilon_{i j} \varepsilon_{i j}
$$

for every symmetric tensor $\varepsilon_{i j}$. (Here and in the sequel of this section we shall use the summation convention for repeated indices.)

Let $\Omega$ be a bounded open domain in $R^{N}$ having a boundary $\partial \Omega$ of class $\mathcal{C}^{2}$. Given a function $\xi: \Omega \longrightarrow \boldsymbol{R}^{N}, \xi(x)=\left(\xi_{1}(x), \cdots, \xi_{N}(x)\right)$, we shall use the notations

$$
\varepsilon_{i j}=\frac{1}{2}\left(\xi_{i, j}+\xi_{j, i}\right) \text { and } \sigma_{i j}=a_{i j k l} \varepsilon_{k l},
$$

where $\xi_{i, j}=\partial \xi_{i} / \partial x_{j}$.

Consider the problem

$$
\begin{gathered}
\xi_{i}^{\prime \prime}-\sigma_{i j, j}+\rho\left(x, \xi_{i}^{\prime}\right)=0 \text { in } \Omega \times \boldsymbol{R}_{+} \\
\xi=0 \text { on } \partial \Omega \times \boldsymbol{R}_{+}
\end{gathered}
$$




$$
\xi(0)=\xi^{0}, \xi^{\prime}(0)=\xi^{1}
$$

Define the energy by

$$
E(t)=\int_{\Omega} \xi_{i}^{\prime} \xi_{i}^{\prime}+\sigma_{i j} \varepsilon_{i j} d x
$$

Assume that (2.5)-(2.10) are satisfied. Then we have the following result:

Theorem 2. Assume that $a \in \mathcal{C}^{0}(\bar{\Omega})$ and is bounded from below by some positive constant $\alpha>0$ on $\omega$ :

$$
\forall x \in \omega, \quad a(x) \geq \alpha>0
$$

Under Hyp. 1 and Hyp. 2, we have:

1. In the simpler case when $g(v)=v$, the energy decays exponentially: there exists a constant $\gamma=\gamma\left(\Omega, \omega, \alpha, \Omega_{j}, x_{j}\right)>0$ such that, given $\left(u^{0}, u^{1}\right) \in H_{0}^{1}(\Omega) \times L^{2}(\Omega)$, the energy of the solution $u$ of $(3.1)-(3.3)$ satisfies the following estimate:

$$
\forall t \geq 0, E(t) \leq E(0) e^{1-\gamma t}
$$

2. In the general case, the energy of the solution $u$ of (2.1)-(2.3) decays as

$$
\forall t>0, E(t) \leq C\left(G^{-1}\left(\frac{1}{t}\right)\right)^{2}
$$

with a constant $C$ only depending on the initial energy $E(0)$ (and in a continuous way).

3. Moreover if $H$ is an increasing function and $H(0)=0$, then in fact

$$
\forall t>0, E(t) \leq C\left(g^{-1}\left(\frac{1}{t}\right)\right)^{2},
$$

with a constant $C$ only depending on the initial energy $E(0)$ (and in a continuous way).

The proof of Theorem 2 is similar to the proof of Theorem 1 .

The proof of Theorem 1 is based on the following integral inequalities that generalize a result from A. Haraux [8]: 


\section{Some nonlinear integral inequalities}

\subsection{The key integral inequality}

Lemma 1. Let $E: \boldsymbol{R}_{+} \longrightarrow \boldsymbol{R}_{+}$be a nonincreasing function and $\phi$ : $\boldsymbol{R}_{+} \longrightarrow \boldsymbol{R}_{+}$a strictly increasing function of class $\mathcal{C}^{1}$ such that

$$
\phi(t) \longrightarrow+\infty \text { as } t \longrightarrow+\infty \text {. }
$$

Assume that there exist $\sigma \geq 0$ and $\omega>0$ such that:

$$
\forall S \geq 1, \int_{S}^{+\infty} E(t)^{1+\sigma} \phi^{\prime}(t) d t \leq \frac{1}{\omega} E(S) .
$$

Then there exists $C>0$ depending on $E(1)$ in a continuous way such that

$$
\begin{gathered}
\text { if } \sigma=0, \text { then } E(t) \leq C e^{-\omega \phi(t)}, \forall t \geq 1 \\
\text { if } \sigma>0, \text { then } E(t) \leq C(\phi(t))^{-1 / \sigma}, \forall t \geq 1 .
\end{gathered}
$$

Proof of Lemma 1. Let us introduce $f:\left[\phi(1),+\infty\left[\longrightarrow \boldsymbol{R}_{+}\right.\right.$defined by

$$
f(\tau)=E\left(\phi^{-1}(\tau)\right)
$$

Then $f$ is nonincreasing and satisfies

$$
\begin{aligned}
\forall 1 \leq S<T<\infty: \int_{\phi(S)}^{\phi(T)} f(\tau)^{1+\sigma} d \tau=\int_{\phi(S)}^{\phi(T)} E\left(\phi^{-1}(\tau)\right)^{1+\sigma} d \tau \\
=\int_{S}^{T} E(t)^{1+\sigma} \phi^{\prime}(t) d t \leq \frac{1}{\omega} E(S)=\frac{1}{\omega} f(\phi(S)) .
\end{aligned}
$$

As $\lim T \rightarrow+\infty \phi(T)=+\infty$, a well-known Gronwall type result (see Komornik [11] p. 124) gives

$$
f(\tau) \leq C e^{-\omega \tau}, \forall \tau \geq \phi(1), \text { if } \sigma=0,
$$

and

$$
f(\tau) \leq C \tau^{-1 / \sigma}, \forall \tau \geq \phi(1), \text { if } \sigma>0,
$$

with $C$ only depending on $f(\phi(1))=E(1)$ and in a continuous way. Since $E(t)=f(\phi(t)),(4.3)$ and $(4.4)$ follow. 


\subsection{Consequences}

First we deduce from (4.4) the following result:

Corollary 4.1. Let $f: \boldsymbol{R}_{+} \longrightarrow \boldsymbol{R}_{+}$be a nonincreasing continuous function.

Assume that there exist $\sigma>0, \sigma^{\prime}>0$ and $c>0$ such that:

$$
\forall t \geq 1, \int_{t}^{+\infty} f(\tau)^{1+\sigma} d \tau \leq c \frac{f(t)}{t^{\sigma^{\prime}}}
$$

Then there exists $C>0$ depending on $f(0)$ in a continuous way such that

$$
\forall t \geq 1, f(t) \leq \frac{C}{t^{\left(1+\sigma^{\prime}\right) / \sigma}}
$$

Proof of Corollary 4.1. Define

$$
g(t)=\frac{f(t)}{t^{\sigma^{\prime}}} \text { if } t \geq 1
$$

$g$ is nonincreasing and satisfies

$$
\forall t \geq 1, \int_{t}^{+\infty} g(\tau)^{1+\sigma} \tau^{\sigma^{\prime}(1+\sigma)} d \tau \leq c g(t) .
$$

Then we can apply Lemma 1 with

$$
\phi(t)=t^{\sigma^{\prime}(1+\sigma)+1}
$$

to deduce that $g$ decays as

$$
g(t) \leq \frac{C}{t^{\left(\sigma^{\prime}(1+\sigma)+1\right) / \sigma}}=\frac{C}{t^{\sigma^{\prime}} t^{\left(1+\sigma^{\prime}\right) / \sigma}} .
$$

So (4.6) follows.

Then we deduce the following integral inequality from Corollary 4.1:

Lemma 2. Let $f: \mathbb{R}_{+} \longrightarrow \mathbb{R}_{+}$be a nonincreasing continuous function. Assume that there exist $\sigma>0, \sigma^{\prime}>0$ and $c>0$ such that:

$$
\forall t \geq 1, \int_{t}^{+\infty} f(\tau)^{1+\sigma} d \tau \leq c f(t)^{1+\sigma}+c \frac{f(t)}{t^{\sigma^{\prime}}} .
$$


Then there exists $C>0$ depending on $f(0)$ in a continuous way such that

$$
\forall t \geq 1, f(t) \leq \frac{C}{t^{\left(1+\sigma^{\prime}\right) / \sigma}}
$$

Proof of Lemma 2. The idea is that the term in $f(t)^{1+\sigma}$ is negligible with respect to the second term. We prove (4.8) using an induction argument. In the following we denote by $C$ all the constants (that depend on $f(0)$ in a continuous way).

First we deduce from (4.7) that

$$
\int_{t}^{+\infty} f(\tau)^{1+\sigma} d \tau \leq C f(t)
$$

Then we deduce from Corollary 4.1 that

$$
f(t) \leq \frac{C}{t^{1 / \sigma}}
$$

Then we use this estimate in (4.7) to deduce that $f$ satisfies

$$
\int_{t}^{+\infty} f(\tau)^{1+\sigma} d \tau \leq C \frac{f(t)}{t}+C \frac{f(t)}{t^{\sigma^{\prime}}}
$$

Define $\sigma_{1}=\inf \left\{1, \sigma^{\prime}\right\}$. Then we have

$$
\int_{t}^{+\infty} f(\tau)^{1+\sigma} d \tau \leq C \frac{f(t)}{t^{\sigma_{1}}}
$$

and we conclude using (4.6) that

$$
f(t) \leq \frac{C}{t^{\left(1+\sigma_{1}\right) / \sigma}} .
$$

If $\sigma^{\prime} \leq 1$ we get (4.8). In the contrary

$$
f(t) \leq \frac{C}{t^{2 / \sigma}}
$$

and we conclude by an obvious induction argument. 
Now we can state the integral inequality that we will use to prove Theorem 1:

Lemma 3. Let $E: \mathbb{R}_{+} \rightarrow \mathbb{R}_{+}$be a nonincreasing function and $\phi:$ $\mathbb{R}_{+} \longrightarrow \mathbb{R}_{+}$a strictly increasing function of class $\mathcal{C}^{1}$ such that

$$
\phi(t) \longrightarrow+\infty \text { as } t \longrightarrow+\infty \text {. }
$$

Assume that there exist $\sigma>0, \sigma^{\prime}>0$ and $c>0$ such that:

$$
\forall S \geq 1, \int_{S}^{+\infty} E(t)^{1+\sigma} \phi^{\prime}(t) d t \leq c E(S)^{1+\sigma}+c \frac{E(S)}{\phi(S)^{\sigma^{\prime}}} .
$$

Then there exists $C>0$ depending on $E(0)$ in a continuous way such that

$$
\forall t \geq 1, E(t) \leq \frac{C}{\phi(t)^{\left(1+\sigma^{\prime}\right) / \sigma}}
$$

Proof of Lemma 3 It is sufficient to introduce

$$
f(\tau)=E\left(\phi^{-1}(\tau)\right)
$$

and to use Lemma 2.

\section{Proof of Theorem 1}

First remark that it is sufficient to prove the estimate (2.15) when the initial conditions verify:

$$
\left(u^{0}, u^{1}\right) \in\left(H^{2}(\Omega) \cup H_{0}^{1}(\Omega)\right) \times H_{0}^{1}(\Omega) .
$$

Then an easy density argument (see for example in Komornik [11] p. $108)$ gives the result for all initial condition in $H_{0}^{1}(\Omega) \times L^{2}(\Omega)$. The regularity given by (2.12) allows us to justify the following computations.

First we verify that (2.1)-(2.3) is a dissipative problem:

\section{Lemma 4.}

$$
\forall 0 \leq S<T<+\infty, \quad E(S)-E(T)=\int_{S}^{T} \int_{\Omega} u^{\prime} \rho\left(x, u^{\prime}\right) d x d t .
$$


Proof of Lemma 4. We multiply (2.1) by $u^{\prime}$ and we integrate by parts on $\Omega \times[S, T]$ :

$$
\begin{aligned}
-\int_{S}^{T} \int_{\Omega} u^{\prime} \rho\left(x, u^{\prime}\right) & =\int_{S}^{T} \int_{\Omega} u^{\prime}\left(u^{\prime \prime}-\Delta u\right)=\left[\frac{1}{2} \int_{\Omega} u^{\prime 2}+|\nabla u|^{2}\right]_{S}^{T} \\
& =E(T)-E(S) .
\end{aligned}
$$

The proof of Theorem 1 is based on the following inequality:

Proposition 5.1. Assume that Hyp. 2 is satisfied. Let $\phi: \boldsymbol{R}_{+} \longrightarrow \boldsymbol{R}$ be a strictly increasing concave function of class $\mathcal{C}^{2}$. Set $\sigma \geq 0$. There exists a positive constant $C$ such that, given $\left(u^{0}, u^{1}\right) \in\left(H^{2}(\Omega) \cup H_{0}^{1}(\Omega)\right) \times$ $H_{0}^{1}(\Omega)$, the solution $u(t)$ of (2.1)-(2.3) satisfies

$$
\begin{aligned}
& \int_{S}^{T} E^{1+\sigma} \phi^{\prime} d \tau \leq C \int_{S}^{T} E^{\sigma} \phi^{\prime} \int_{\omega}{u^{\prime}}^{2} d x d \tau \\
& +C \int_{S}^{T} E^{\sigma} \phi^{\prime} \int_{\Omega} \rho\left(x, u^{\prime}\right)^{2} d x d \tau+C E(S)^{1+\sigma}
\end{aligned}
$$

Remarks. 1. The proof of (5.3) is based on multiplier techniques; the constant $C$ will be explicit.

2. When $\phi^{\prime}(t)=1,(5.3)$ is classical. E. Zuazua [23], M. Nakao [19] and L.R. Tcheugoué Tébou [21] proved it under different hypothesis on the term $\rho(x, \cdot)$ when $\omega$ contains a neighborhood of $\Gamma\left(x^{0}\right) ; K$. Liu and M. Yamamoto [15] proved it under Hyp. 2 with $\rho\left(x, u^{\prime}\right)=0$ and $\sigma=0$. The term $\phi^{\prime}(t)$ will be essential to obtain a precise estimate on the decay rate of the energy when we do not make any assumption on the behavior of $g$ near 0 . In Section 6 we will show how to define $\phi: \phi$ will be closely related on the behavior of $\rho(x, \cdot)$ and will allow us to eliminate the usual assumption of polynomial growth of $\rho(x, \cdot)$ in zero.

In the following computations, leading to prove (5.3), we omit to write the differential elements in order to simplify the expressions. All the constants $C$ will be explicit.

\subsection{The piecewise multiplier method}

Lemma 5. Let $\mathcal{O} \subset \Omega$ be a Lipschitz domain. 
Let $h: \mathcal{O} \longrightarrow \mathbb{R}^{N}$ be a vector field of class $\mathcal{C}^{1}$. Let $\phi: \mathbb{R}_{+} \longrightarrow \mathbb{R}_{+} a$ function of class $\mathcal{C}^{2}$. Set $0 \leq S<T<+\infty$ and $\sigma \geq 0$. Then we have the following identity:

$$
\begin{aligned}
& \int_{S}^{T} E^{\sigma} \phi^{\prime} \int_{\partial \mathcal{O}} 2 \partial_{\nu} u h \cdot \nabla u+(h \cdot \nu)\left(u^{\prime 2}-|\nabla u|^{2}\right) \\
& =\left[E^{\sigma} \phi^{\prime} \int_{\mathcal{O}} 2 u^{\prime} h \cdot \nabla u\right]_{S}^{T}-\int_{S}^{T}\left(\sigma E^{\prime} E^{\sigma-1} \phi^{\prime}+E^{\sigma} \phi^{\prime \prime}\right) \int_{\mathcal{O}} 2 u^{\prime} h \cdot \nabla u \\
& +\int_{S}^{T} E^{\sigma} \phi^{\prime} \int_{\mathcal{O}}(\operatorname{div} h)\left(u^{\prime 2}-|\nabla u|^{2}\right) \\
& +2 \sum_{i, k} \frac{\partial h_{k}}{\partial x_{i}} \frac{\partial u}{\partial x_{i}} \frac{\partial u}{\partial x_{k}}+2 \rho\left(x, u^{\prime}\right) h \cdot \nabla u .
\end{aligned}
$$

Remark. The integrations by part that lead to (5.4) can usually be done if $\mathcal{O}$ is of class $\mathcal{C}^{2}$, and remain valid if $\mathcal{O}$ is only Lipschitz thanks to the results of $P$. Grisvard [6].

Proof of Lemma 5. This identity is given by the multiplier method: integrate by parts the following expression:

$$
\begin{aligned}
& 0=\int_{S}^{T} E^{\sigma} \phi^{\prime} \int_{\mathcal{O}} 2 h \cdot \nabla u\left(u^{\prime \prime}-\Delta u+\rho\left(x, u^{\prime}\right)\right) \\
= & {\left[E^{\sigma} \phi^{\prime} \int_{\mathcal{O}} 2 u^{\prime} h \cdot \nabla u\right]_{S}^{T}-\int_{S}^{T}\left(\sigma E^{\prime} E^{\sigma-1} \phi^{\prime}+E^{\sigma} \phi^{\prime \prime}\right) \int_{\mathcal{O}} 2 u^{\prime} h \cdot \nabla u } \\
& -\int_{S}^{T} E^{\sigma} \phi^{\prime} \int_{\mathcal{O}} 2 u^{\prime} h \cdot \nabla u^{\prime}-\int_{S}^{T} E^{\sigma} \phi^{\prime} \int_{\partial \mathcal{O}} 2 \partial_{\nu} u h \cdot \nabla u \\
+ & \int_{S}^{T} E^{\sigma} \phi^{\prime} \int_{\mathcal{O}} \nabla u \cdot \nabla(2 h \cdot \nabla u)+2 \rho\left(x, u^{\prime}\right) h \cdot \nabla u \\
= & {\left[E^{\sigma} \phi^{\prime} \int_{\mathcal{O}} 2 u^{\prime} h \cdot \nabla u\right]_{S}^{T}-\int_{S}^{T}\left(\sigma E^{\prime} E^{\sigma-1} \phi^{\prime}+E^{\sigma} \phi^{\prime \prime}\right) \int_{\mathcal{O}} 2 u^{\prime} h \cdot \nabla u } \\
- & \int_{S}^{T} E^{\sigma} \phi^{\prime} \int_{\partial \mathcal{O}} 2 \partial_{\nu} u h \cdot \nabla u+h \cdot \nu u^{\prime 2}+\int_{S}^{T} E^{\sigma} \phi^{\prime} \int_{\mathcal{O}}(\operatorname{div} h) u^{\prime 2} \\
+ & \int_{S}^{T} E^{\sigma} \phi^{\prime} \int_{\mathcal{O}} 2 \sum_{i, k}\left(\partial_{i} h_{k} \partial_{i} u \partial_{k} u+h_{k} \partial_{i} u \partial_{i, k}^{2} u\right)+2 \rho\left(x, u^{\prime}\right) h \cdot \nabla u
\end{aligned}
$$




$$
\begin{aligned}
& =\left[E^{\sigma} \phi^{\prime} \int_{\mathcal{O}} 2 u^{\prime} h \cdot \nabla u\right]_{S}^{T}-\int_{S}^{T}\left(\sigma E^{\prime} E^{\sigma-1} \phi^{\prime}+E^{\sigma} \phi^{\prime \prime}\right) \int_{\mathcal{O}} 2 u^{\prime} h \cdot \nabla u \\
& -\int_{S}^{T} E^{\sigma} \phi^{\prime} \int_{\partial \mathcal{O}} 2 \partial_{\nu} u h \cdot \nabla u+h \cdot \nu\left(u^{\prime 2}-|\nabla u|^{2}\right) \\
& +\int_{S}^{T} E^{\sigma} \phi^{\prime} \int_{\mathcal{O}}(\operatorname{div} h)\left(u^{\prime 2}-|\nabla u|^{2}\right) \\
& +2 \sum_{i, k} \partial_{i} h_{k} \partial_{i} u \partial_{k} u+2 \rho\left(x, u^{\prime}\right) h \cdot \nabla u
\end{aligned}
$$

So, putting the boundary integrals in the left-hand side, we get (5.4).

The main problem is to estimate, and more precisely to majorate, the boundary integrals in (5.4). Usually this kind of identity is used with $\mathcal{O}=\Omega$ and an adequate vector field $h$ so that the boundary condition allows us to estimate the boundary integral: this can be easily done on $\{u=0\} \cap\{m \cdot \nu \leq 0\}$, and $h$ is chosen so that it is equal to zero on the other part of the boundary.

In our case, we will use this identity on each domain $\Omega_{j}$. In order to avoid the problems given by the lack of information about the values of $u$ on each $\partial \Omega_{j}$, Liu [14] constructed a special vector field $h_{j}$ that allows us to use the same strategy as before: one of its properties is to be equal to zero on $\left(\partial \Omega_{j} \backslash \partial \Omega\right) \cup \Gamma_{j}\left(x_{j}\right)$. We describe the construction of $h_{j}$ : set $0<\varepsilon_{0}<\varepsilon_{1}<\varepsilon_{2}<\varepsilon$ and define for $i=0$ to 2 :

$$
Q_{i}=\mathcal{N}_{e_{i}}\left[\cup_{j} \Gamma_{j}\left(x_{j}\right) \cup\left(\Omega \backslash \cup_{j} \Omega_{j}\right)\right]
$$

Since $\left(\overline{\Omega_{j}} \backslash Q_{1}\right) \cap \overline{Q_{0}}=\emptyset$, we can construct a function $\psi_{j} \in \mathcal{C}_{0}^{\infty}\left(\boldsymbol{R}^{N}\right)$ that satisfies:

$$
\begin{gathered}
0 \leq \psi_{j} \leq 1, \\
\psi_{j}=1 \text { on } \overline{\Omega_{j}} \backslash Q_{1}, \\
\psi_{j}=0 \text { on } Q_{0} .
\end{gathered}
$$

Now we use the identity (5.4) with $\mathcal{O}:=\Omega_{j}$ and $h=h_{j}(x):=\psi_{j}(x) m_{j}(x)$ :

$$
\int_{S}^{T} E^{\sigma} \phi^{\prime} \int_{\partial \Omega_{j}} 2 \partial_{\nu} u \psi_{j} m_{j} \cdot \nabla u+\left(\psi_{j} m_{j} \cdot \nu\right)\left(u^{\prime 2}-|\nabla u|^{2}\right)
$$




$$
\begin{gathered}
=\left[E^{\sigma} \phi^{\prime} \int_{\Omega_{j}} 2 u^{\prime} \psi_{j} m_{j} \cdot \nabla u\right]_{S}^{T}-\int_{S}^{T}\left(\sigma E^{\prime} E^{\sigma-1} \phi^{\prime}+E^{\sigma} \phi^{\prime \prime}\right) \int_{\Omega_{j}} 2 u^{\prime} \psi_{j} m_{j} \cdot \nabla u \\
+\int_{S}^{T} E^{\sigma} \phi^{\prime} \int_{\Omega_{j}}\left(\operatorname{div} \psi_{j} m_{j}\right)\left(u^{\prime 2}-|\nabla u|^{2}\right) \\
+2 \sum_{i, k} \frac{\partial\left(\psi_{j} m_{j}\right)_{k}}{\partial x_{i}} \frac{\partial u}{\partial x_{i}} \frac{\partial u}{\partial x_{k}}+2 \rho\left(x, u^{\prime}\right) \psi_{j} m_{j} \cdot \nabla u
\end{gathered}
$$

First we look at the boundary integral in (5.9). Because of its construction, $\psi_{j}=0$ outside $\left(\left(\partial \Omega_{j} \backslash \Gamma_{j}\left(x_{j}\right)\right) \cap \partial \Omega\right)$. So only the part on $\left(\left(\partial \Omega_{j} \backslash \Gamma_{j}\left(x_{j}\right)\right) \cap \partial \Omega\right)$ has to be taken in count. But on this part, $u=0$, so $u^{\prime}=0$ and $\nabla u=\left(\partial_{\nu} u\right) \nu=\left(\partial_{\nu_{j}} u\right) \nu_{j}$. Thus the boundary integral in (5.9) is equal to

$$
\begin{aligned}
& \int_{S}^{T} E^{\alpha} \phi^{\prime} \int_{\left(\left(\partial \Omega_{j} \backslash \Gamma_{j}\left(x_{j}\right)\right) \cap \partial \Omega\right)} 2 \partial_{\nu_{j}} u \psi_{j} m_{j} \cdot \nabla u+\left(\psi_{j} m_{j} \cdot \nu_{j}\right)\left(u^{\prime 2}-|\nabla u|^{2}\right) \\
& =\int_{S}^{T} E^{\sigma} \phi^{\prime} \int_{\left(\left(\partial \Omega_{j} \backslash \Gamma_{j}\left(x_{j}\right)\right) \cap \partial \Omega\right)} \psi_{j}\left(m_{j} \cdot \nu_{j}\right)\left(\partial_{\nu_{j}} u\right)^{2} \leq 0 .
\end{aligned}
$$

So, using (5.8), we deduce from (5.9) that we have for each index $j$

$$
\begin{gathered}
{\left[E^{\sigma} \phi^{\prime} \int_{\Omega_{j}} 2 u^{\prime} \psi_{j} m_{j} \cdot \nabla u\right]_{S}^{T}-\int_{S}^{T}\left(\sigma E^{\prime} E^{\sigma-1} \phi^{\prime}+E^{\sigma} \phi^{\prime \prime}\right) \int_{\Omega_{j}} 2 u^{\prime} \psi_{j} m_{j} \cdot \nabla u} \\
+\int_{S}^{T} E^{\sigma} \phi^{\prime} \int_{\Omega_{j} \backslash Q_{0}}\left(\operatorname{div} \psi_{j} m_{j}\right)\left(u^{\prime 2}-|\nabla u|^{2}\right) \\
+\int_{S}^{T} E^{\sigma} \phi^{\prime} \int_{\Omega_{j} \backslash Q_{0}} 2 \sum_{i, k} \frac{\partial\left(\psi_{j} m_{j}\right)_{k}}{\partial x_{i}} \frac{\partial u}{\partial x_{i}} \frac{\partial u}{\partial x_{k}}+2 \rho\left(x, u^{\prime}\right) \psi_{j} m_{j} \cdot \nabla u \leq 0
\end{gathered}
$$

Thanks to (5.7), we have

$$
\begin{aligned}
& \sum_{j}\left[E^{\sigma} \phi^{\prime} \int_{\Omega_{j}} 2 u^{\prime} \psi_{j} m_{j} \cdot \nabla u\right]_{S}^{T} \\
& -\sum_{j} \int_{S}^{T}\left(\sigma E^{\prime} E^{\sigma-1} \phi^{\prime}+E^{\sigma \sigma} \phi^{\prime \prime}\right) \int_{\Omega_{j}} 2 u^{\prime} \psi_{j} m_{j} \cdot \nabla u \\
& +\int_{S}^{T} E^{\sigma} \phi^{\prime} \int_{\Omega_{j} \backslash Q_{1}} N u^{\prime 2}+(2-N)|\nabla u|^{2}
\end{aligned}
$$


A new method to obtain decay rate estimates for...

$$
\begin{aligned}
& +\sum_{j} \int_{S}^{T} E^{\sigma} \phi^{\prime} \int_{\Omega_{j}} 2 \rho\left(x, u^{\prime}\right) \psi_{j} m_{j} \cdot \nabla u \\
& \leq-\sum_{j} \int_{S}^{T} E^{\sigma} \phi^{\prime} \int_{\Omega_{j} \cap Q_{1}}\left(\operatorname{div} \psi_{j} m_{j}\right)\left(u^{\prime 2}-|\nabla u|^{2}\right) \\
& +2 \sum_{i, k} \frac{\partial\left(\psi_{j} m_{j}\right)_{k}}{\partial x_{i}} \frac{\partial u}{\partial x_{i}} \frac{\partial u}{\partial x_{k}} \\
& \leq C \sum_{j} \int_{S}^{T} E^{\sigma} \phi^{\prime} \int_{\Omega_{j} \cap Q_{1}} u^{\prime 2}+|\nabla u|^{2} \\
& \leq C \int_{S}^{T} E^{\sigma} \phi^{\prime} \int_{\Omega_{n} Q_{i}} u^{\prime 2}+|\nabla u|^{2},
\end{aligned}
$$

where $C=C(\varphi, m)$ is a positive constant that only depends on $\varphi_{j}$ and $m_{j}$. (Note that $\cup_{j}\left(\Omega_{j} \backslash Q_{1}\right)=\Omega \backslash Q_{1}$.)

As usually, we obtain another key identity multiplying $(2.1)$ by $(N-1) u$ :

$$
(N-1) \int_{S}^{T} E^{\sigma} \phi^{\prime} \int_{\Omega} u\left(u^{\prime \prime}-\Delta u+\rho\left(x, u^{\prime}\right)\right)=0,
$$

so

$$
\begin{aligned}
& (N-1)\left[E^{\sigma} \phi^{\prime} \int_{\Omega} u u^{\prime}\right]_{S}^{T}-(N-1) \int_{S}^{T}\left(\sigma E^{\prime} E^{\sigma-1} \phi^{\prime}+E^{\sigma} \phi^{\prime \prime}\right) \int_{\Omega} u u^{\prime} \\
& +(N-1) \int_{S}^{T} E^{\sigma} \phi^{\prime} \int_{\Omega}|\nabla u|^{2}-u^{\prime 2}+u \rho\left(x, u^{\prime}\right)=0
\end{aligned}
$$

Denote

$$
\left\{\begin{array}{l}
h(x):=\psi_{j}(x) m_{j}(x) \text { if } x \in \Omega_{j} \\
h(x):=0 \text { if } x \in \Omega \backslash \cup_{j} \Omega_{j}
\end{array}\right.
$$

and

$$
M(u):=2 h \cdot \nabla u+(N-1) u .
$$

We add (5.11) to (5.10) to obtain:

$$
\begin{aligned}
& {\left[E^{\sigma} \phi^{\prime} \int_{\Omega} M(u) u^{\prime}\right]_{S}^{T}-\int_{S}^{T}\left(\sigma E^{\prime} E^{\sigma-1} \phi^{\prime}+E^{\sigma} \phi^{\prime \prime}\right)\left(\int_{\Omega} M(u) u^{\prime}\right)} \\
& +\int_{S}^{T} E^{\sigma} \phi^{\prime} \int_{\Omega} M(u) \rho\left(x, u^{\prime}\right)+\int_{S}^{T} E^{\sigma} \phi^{\prime} \int_{\Omega_{j} \backslash Q_{1}} u^{\prime 2}+|\nabla u|^{2} \\
& \leq C^{\prime} \int_{S}^{T} E^{\sigma} \phi^{\prime} \int_{\Omega \cap Q_{1}} u^{\prime 2}+|\nabla u|^{2}
\end{aligned}
$$


So

$$
\begin{aligned}
& 2 \int_{S}^{T} E^{1+\sigma} \phi^{\prime} \leq\left(C^{\prime}+1\right) \int_{S}^{T} E^{\sigma} \phi^{\prime} \int_{\Omega \cap Q_{1}} u^{\prime 2}+|\nabla u|^{2} \\
& -\left[E^{\sigma} \phi^{\prime} \int_{\Omega} M(u) u^{\prime}\right]_{S}^{T}+\int_{S}^{T}\left(\sigma E^{\prime} E^{\sigma-1} \phi^{\prime}+E^{\sigma} \phi^{\prime \prime}\right)\left(\int_{\Omega} M(u) u^{\prime}\right) \\
& -\int_{S}^{T} E^{\sigma} \phi^{\prime} \int_{\Omega} M(u) \rho\left(x, u^{\prime}\right)
\end{aligned}
$$

This is the key inequality to prove (5.3). Next we estimate the different terms of the right-hand side member.

\subsection{Estimates of the right-hand side terms of (5.13)}

Let $\phi$ be an increasing concave function of class $\mathcal{C}^{2}$ on $\boldsymbol{R}_{+}$.

Lemma 6. There exists a positive constant $C$ such that

$$
\left|E^{\sigma} \phi^{\prime} \int_{\Omega} M(u) u^{\prime} d x\right| \leq C E(t)^{1+\sigma} .
$$

Proof of Lemma 6. By hypothesis $\phi^{\prime}$ is a nonnegative and nonincreasing function. So $\phi^{\prime}$ is bounded on $\mathbb{R}_{+}$. Since there exists $c>0$ such that

$$
\int_{\Omega} M(u)^{2} d x \leq c E(t)
$$

we have

$$
\left|\int_{\Omega} M(u) u^{\prime} d x\right| \leq c^{\prime} E(t),
$$

and so (5.14) holds.

Lemma 7. There exists a positive constant $C$ such that

$$
\left|\int_{S}^{T}\left(\sigma E^{\prime} E^{\sigma-1} \phi^{\prime}+E^{\sigma} \phi^{\prime \prime}\right)\left(\int_{\Omega} M(u) u^{\prime} d x\right) d \tau\right| \leq C E(S)^{1+\sigma}
$$


Proof of Lemma 7. Since $\phi$ is concave and $E$ is nonincreasing, we have

$$
\begin{aligned}
& \left|\int_{S}^{T}\left(\sigma E^{\prime} E^{\sigma-1} \phi^{\prime}+E^{\sigma} \phi^{\prime \prime}\right)\left(\int_{\Omega} M(u) u^{\prime} d x\right)\right| \\
& \quad \leq \int_{S}^{T}\left|\sigma E^{\prime} E^{\sigma-1} \phi^{\prime}+E^{\sigma} \phi^{\prime \prime}\right| c E(\tau) d \tau \\
& \quad \leq c \int_{S}^{T}-E^{\prime}(\tau) E(\tau)^{\sigma} d \tau+c E(S)^{1+\sigma} \int_{S}^{T}-\phi^{\prime \prime}(\tau) d \tau \\
& \quad \leq \frac{c}{1+\sigma} E(S)^{1+\sigma}+c \phi^{\prime}(S) E(S)^{1+\sigma} .
\end{aligned}
$$

Lemma 8. There exists a positive constant $C$ such that for all $\delta>0$ we have

$$
\begin{aligned}
& \left|\int_{S}^{T} E^{\sigma} \phi^{\prime} \int_{\Omega} M(u) \rho\left(x, u^{\prime}\right) d x d \tau\right| \\
& \quad \leq \frac{C}{\delta} \int_{S}^{T} E^{\sigma} \phi^{\prime} \int_{\Omega} \rho\left(x, u^{\prime}\right)^{2} d x d \tau+\delta \int_{S}^{T} E^{1+\sigma} \phi^{\prime} d \tau .
\end{aligned}
$$

Proof of Lemma 8. Set $\delta>0$.

$$
\begin{aligned}
& \left|\int_{S}^{T} E^{\sigma} \phi^{\prime} \int_{\Omega} M(u) \rho\left(x, u^{\prime}\right)\right| \leq \int_{S}^{T} E^{\sigma} \phi^{\prime} \int_{\Omega} \frac{\delta}{2} M(u)^{2}+\frac{1}{2 \delta} \rho\left(x, u^{\prime}\right)^{2} \\
& \quad \leq \frac{c \delta}{2} \int_{S}^{T} E^{1+\sigma} \phi^{\prime}+\frac{1}{2 \delta} \int_{S}^{T} E^{\sigma} \phi^{\prime} \int_{\Omega} \rho\left(x, u^{\prime}\right)^{2}
\end{aligned}
$$

Using the estimates (5.14)-(5.16) with $\delta$ small enough, we deduce from (5.13) that there exists a positive constant $C$ such that

$$
\begin{aligned}
& \int_{S}^{T} E^{1+\sigma} \phi^{\prime} d \tau \leq C E(S)^{1+\sigma}+C \int_{S}^{T} E^{\sigma} \phi^{\prime} \int_{\Omega} \rho\left(x, u^{\prime}\right)^{2} d x d \tau \\
& \quad+C \int_{S}^{T} E^{\sigma} \phi^{\prime} \int_{\Omega Q_{1}} u^{\prime 2}+|\nabla u|^{2} d x d \tau
\end{aligned}
$$

It remains to estimate the last term. 
Lemma 9. There exists a positive constant $C$ such that, for all $\delta>0$ :

$$
\begin{aligned}
& \int_{S}^{T} E^{\sigma} \phi^{\prime} \int_{\Omega \cap Q_{1}}|\nabla u|^{2} d x d \tau \leq C E(S)^{1+\sigma} \\
& +C \int_{S}^{T} E^{\sigma} \phi^{\prime} \int_{\Omega \cap Q_{2}} u^{\prime 2}+\rho\left(x, u^{\prime}\right)^{2}+u^{2} d x d \tau .
\end{aligned}
$$

Proof of Lemma $\boldsymbol{\theta}$. Since $\overline{\boldsymbol{R}^{N} \backslash \overline{Q_{2}}} \cap \overline{Q_{1}}=\emptyset$, we can construct $\xi \epsilon$ $\mathcal{C}_{0}^{\infty}\left(\boldsymbol{R}^{N}\right)$ that satisfies

$$
\left\{\begin{array}{l}
0 \leq \xi \leq 1 \\
\xi=1 \text { on } Q_{1} \\
\xi=0 \text { on } \mathbb{R}^{N} \backslash Q_{2} .
\end{array}\right.
$$

We multiply (2.1) by $\xi u$ to obtain:

$$
\begin{aligned}
& \int_{S}^{T} E^{\sigma} \phi^{\prime} \int_{\Omega}-\xi u \rho\left(x, u^{\prime}\right)=\int_{S}^{T} E^{\sigma} \phi^{\prime} \int_{\Omega} \xi u\left(u^{\prime \prime}-\Delta u\right) \\
& =\left[E^{\sigma} \phi^{\prime} \int_{\Omega} \xi u u^{\prime}\right]_{S}^{T}-\int_{S}^{T}\left(\sigma E^{\prime} E^{\sigma-1} \phi^{\prime}+E^{\sigma} \phi^{\prime \prime}\right) \int_{\Omega} \xi u u^{\prime} \\
& +\int_{S}^{T} E^{\sigma} \phi^{\prime} \int_{\Omega}-\xi u^{\prime 2}+\nabla u \cdot \nabla(\xi u) \\
& =\left[E^{\sigma} \phi^{\prime} \int_{\Omega} \xi u u^{\prime}\right]_{S}^{T}-\int_{S}^{T}\left(\sigma E^{\prime} E^{\sigma-1} \phi^{\prime}+E^{\sigma} \phi^{\prime \prime}\right) \int_{\Omega} \xi u u^{\prime} \\
& +\int_{S}^{T} E^{\sigma} \phi^{\prime} \int_{\Omega} \xi\left(|\nabla u|^{2}-u^{\prime 2}\right)-\frac{1}{2} u^{2} \Delta \xi .
\end{aligned}
$$

We deduce that

$$
\begin{aligned}
& \int_{S}^{T} E^{\sigma} \phi^{\prime} \int_{\Omega \cap Q_{1}}|\nabla u|^{2} \leq-\left[E^{\sigma} \phi^{\prime} \int_{\Omega} \xi u u^{\prime}\right]_{S}^{T} \\
& +\int_{S}^{T}\left(\sigma E^{\prime} E^{\sigma-1} \phi^{\prime}+E^{\sigma} \phi^{\prime \prime}\right) \int_{\Omega} \xi u u^{\prime} \\
& +\int_{S}^{T} E^{\sigma} \phi^{\prime} \int_{\Omega}-\xi u \rho\left(x, u^{\prime}\right)+\xi u^{\prime 2}+\frac{1}{2} u^{2} \Delta \xi \\
& \leq C E(S)^{1+\sigma}+C \int_{S}^{T} E^{\sigma} \phi^{\prime} \int_{\Omega Q_{2}} \rho\left(x, u^{\prime}\right)^{2}+u^{\prime 2}+u^{2}
\end{aligned}
$$


At last we adapt a method introduced in Conrad and Rao [4] to estimate the last term of (5.18):

Lemma 10. There exists a positive constant $C$ such that, for all $\eta>0$ :

$$
\begin{aligned}
& \int_{S}^{T} E^{\sigma} \phi^{\prime} \int_{\Omega \mathrm{n} Q_{2}} u^{2} d x d \tau \leq C E(S)^{1+\sigma}+\eta \int_{S}^{T} E^{1+\sigma} \phi^{\prime} d \tau \\
& +\frac{C}{\eta} \int_{S}^{T} E^{\sigma} \phi^{\prime} \int_{\Omega} \rho\left(x, u^{\prime}\right)^{2} d x d \tau+\frac{C}{\eta} \int_{S}^{T} E^{\sigma} \phi^{\prime} \int_{\omega} u^{\prime 2} d x d \tau
\end{aligned}
$$

Proof of Lemma 10. Since $\overline{\boldsymbol{R}^{N} \backslash \omega} \cap \overline{Q_{2}}=\emptyset$, we can construct $\beta \in$ $\mathcal{C}_{0}^{\infty}\left(\boldsymbol{R}^{N}\right)$ that satisfies

$$
\left\{\begin{array}{l}
0 \leq \beta \leq 1_{t} \\
\beta=1 \text { on } Q_{2} \\
\beta=0 \text { on } R^{N} \backslash \omega
\end{array}\right.
$$

Fix $t$ and consider the solution $z$ of the following elliptic problem:

$$
\begin{gathered}
\Delta z=\beta(x) u \text { in } \Omega, \\
z=0 \text { on } \partial \Omega .
\end{gathered}
$$

We multiply (5.20) by $z$ to deduce that there exists $c>0$ such that:

$$
\int_{\Omega}|\nabla z|^{2}=-\int_{\Omega} \beta u z \leq c\|u\|_{L^{2}(\Omega)}\|z\|_{L^{2}(\Omega)} .
$$

So

$$
\|z\|_{L^{2}(\Omega)} \leq c\|u\|_{L^{2}(\Omega)} .
$$

On the other hand, deriving with respect to $t$, we see that $z^{\prime}$ is solution of the problem:

$$
\begin{gathered}
\Delta z^{\prime}=\beta(x) u^{\prime} \text { in } \Omega, \\
z^{\prime}=0 \text { on } \partial \Omega .
\end{gathered}
$$

So we deduce that

$$
\int_{\Omega} z^{\prime 2} \leq c \int_{\Omega} \beta u^{\prime 2}
$$


Next we multiply $(2.1)$ by $z$ :

$$
\begin{aligned}
0 & =\int_{S}^{T} E^{\sigma} \phi^{\prime} \int_{\Omega} z\left(u^{\prime \prime}-\Delta u+\rho\left(x, u^{\prime}\right)\right) \\
& =\left[E^{\sigma} \phi^{\prime} \int_{\Omega} z u^{\prime}\right]_{S}^{T}-\int_{S}^{T}\left(\sigma E^{\prime} E^{\sigma-1} \phi^{\prime}+E^{\sigma} \phi^{\prime \prime}\right) \int_{\Omega} z u^{\prime} \\
& +\int_{S}^{T} E^{\sigma} \phi^{\prime} \int_{\Omega}-z^{\prime} u^{\prime}-u \Delta z+z \rho\left(x, u^{\prime}\right)
\end{aligned}
$$

So

$$
\begin{aligned}
& \int_{S}^{T} E^{\sigma} \phi^{\prime} \int_{\Omega} \beta u^{2}=\left[E^{\sigma} \phi^{\prime} \int_{\Omega} z u^{\prime}\right]_{S}^{T}-\int_{S}^{T}\left(\sigma E^{\prime} E^{\sigma-1} \phi^{\prime}+E^{\sigma} \phi^{\prime \prime}\right) \int_{\Omega} z u^{\prime} \\
& +\int_{S}^{T} E^{\sigma} \phi^{\prime} \int_{\Omega}-z^{\prime} u^{\prime}+z \rho\left(x, u^{\prime}\right)
\end{aligned}
$$

First we note that

$$
\left|\left[E^{\sigma} \phi^{\prime} \int_{\Omega} z u^{\prime}\right]_{S}^{T}-\int_{S}^{T}\left(\sigma E^{\prime} E^{\sigma-1} \phi^{\prime}+E^{\sigma} \phi^{\prime \prime}\right) \int_{\Omega} z u^{\prime}\right| \leq c E(S)^{1+\sigma} .
$$

Next set $\eta>0$ :

$$
\begin{aligned}
& \left|\int_{S}^{T} E^{\sigma} \phi^{\prime} \int_{\Omega} z^{\prime} u^{\prime}\right| \leq \int_{S}^{T} E^{\sigma} \phi^{\prime} c\left(\int_{\Omega} \beta u^{\prime 2}\right)^{1 / 2}\left(\int_{\Omega} u^{\prime 2}\right)^{1 / 2} \\
& \quad \leq \frac{c}{2 \eta} \int_{S}^{T} E^{\sigma} \phi^{\prime} \int_{\Omega} \beta u^{\prime 2}+\frac{c \eta}{2} \int_{S}^{T} E^{\sigma} \phi^{\prime} \int_{\Omega} u^{\prime 2} \\
& \quad \leq \frac{c}{\eta} \int_{S}^{T} E^{\sigma} \phi^{\prime} \int_{\omega} u^{\prime 2}+c \eta \int_{S}^{T} E^{1+\sigma} \phi^{\prime} .
\end{aligned}
$$

The last term can be treated in the same way:

$$
\left|\int_{S}^{T} E^{\sigma} \phi^{\prime} \int_{\Omega} z \rho\left(x, u^{\prime}\right)\right| \leq \frac{c}{\eta} \int_{S}^{T} E^{\sigma} \phi^{\prime} \int_{\Omega} \rho\left(x, u^{\prime}\right)^{2}+\eta c \int_{S}^{T} E^{1+\sigma} \phi^{\prime} .
$$

We deduce from (5.26) and from the last three estimates the validity of (5.19). 


\subsection{End of the proof of Proposition 5.1.}

Now we can achieve the proof of Proposition 5.1: using (5.18) and then (5.19), we deduce from (5.17) that there exists $C>0$ such that for all $\eta>0$

$$
\begin{aligned}
& \int_{S}^{T} E^{1+\sigma} \phi^{\prime} d \tau \leq C E(S)^{1+\sigma}+\frac{C}{\eta} \int_{S}^{T} E^{\sigma} \phi^{\prime} \int_{\Omega} \rho\left(x, u^{\prime}\right)^{2} d x d \tau \\
& +\frac{C}{\eta} \int_{S}^{T} E^{\sigma} \phi^{\prime} \int_{\omega}{u^{\prime}}^{2} d x d \tau+\eta \int_{S}^{T} E^{1+\sigma} \phi^{\prime} d \tau
\end{aligned}
$$

So if we choose $\eta<1$, we get (5.3).

\section{Estimates on the decay rate of the energy}

When $\rho(x, \cdot)$ has a polynomial growth in 0 , the last two terms of (5.3) can be estimated using the Hölder inequality (see e.g. V. Komornik [11]). But when we don't make any assumption on the growth of $\rho(x, \cdot)$ near 0 , we cannot apply the same reasoning. The information we need on the growth of $\rho(x, \cdot)$ will be contained in the behavior of $\phi$ at infinity. The function $\phi$ will be carefully chosen so that we can apply Lemma 3 .

\subsection{Exponential decay of the energy if $g(v)=v$}

We set in the case where

$$
\forall x \in \Omega, v \in \mathbb{R}, a(x)|v| \leq|\rho(x, v)| \leq C a(x)|v| .
$$

Then we show that the energy decays exponentially: since $a(x) \geq \alpha>0$ on $\omega$, we deduce from Proposition 5.1 that

$$
\begin{aligned}
& \int_{S}^{T} E^{1+\sigma} \phi^{\prime} d \tau \leq C E^{1+\sigma}(S)+C \int_{S}^{T} E^{\sigma} \phi^{\prime} \int_{\Omega} a u^{\prime 2} d x d \tau \\
& \leq C E^{1+\sigma}(S)+C \int_{S}^{T} E^{\sigma} \phi^{\prime} \int_{\Omega} u^{\prime} \rho\left(x, u^{\prime}\right) d x d \tau
\end{aligned}
$$

Choosing $\phi(t)=t$ and $\sigma=0$, we deduce from (6.1) and from (5.2) that

$$
\int_{S}^{T} E(\tau) d \tau \leq C E(S)+C \int_{S}^{T} \int_{\Omega} u^{\prime} \rho\left(x, u^{\prime}\right) d x d \tau=2 C E(S)
$$


Then the well-known Gronwall type inequality (4.3) gives us that

$$
E(t) \leq C^{\prime} e^{-t /(2 C)} \text {. }
$$

(Note that it is easy to see that $C^{\prime}=e E(0)$.) This achieves the proof of the first part of Theorem 1 .

\subsection{General estimate on the decay rate: proof of (2.15)}

Assume that Hyp. 1 is satisfied with some strictly increasing odd function $g$ of class $\mathcal{C}^{1}$. Fix $\sigma=1$. Assume now that $\phi$ is a strictly increasing concave function of class $\mathcal{C}^{2}$ such that

$$
\phi(t) \longrightarrow+\infty \text { and } \phi^{\prime}(t) \longrightarrow 0 \text { as } t \longrightarrow+\infty
$$

(for example $t \mapsto \ln (1+t)$ ).

We estimate the terms of the right-hand side of $(5.3)$ in order to apply the results of Lemma 3: first we look at

$$
\int_{S}^{T} E \phi^{\prime} \int_{\omega} u^{\prime 2} d x d t
$$

We have the following estimate

Lemma 11. There exists $C>0$ such that

$$
\int_{S}^{T} E \phi^{\prime} \int_{\omega} u^{\prime 2} d x d t \leq C E(S)^{2}+C E(S) \int_{S}^{T} \phi^{\prime}(t)\left(g^{-1}\left(\phi^{\prime}(t)\right)\right)^{2} d t .
$$

Proof of Lemma 11. Let us introduce

$$
h(t)=g^{-1}\left(\phi^{\prime}(t)\right)
$$

$h$ is a decreasing positive function and satisfies

$$
h(t) \longrightarrow 0 \text { as } t \longrightarrow+\infty .
$$

Fix $t \geq 1$ and define

$$
\Omega_{1,1}^{t}:=\left\{x \in \Omega:\left|u^{\prime}\right| \leq h(t)\right\},
$$




$$
\begin{gathered}
\Omega_{1,2}^{t}:=\left\{x \in \Omega: h(t)<\left|u^{\prime}\right| \leq h(1)\right\}, \\
\Omega_{1,3}^{t}:=\left\{x \in \Omega:\left|u^{\prime}\right|>h(1)\right\} .
\end{gathered}
$$

Fix $S \geq 1$; first we look at the part on $\Omega_{1,3}^{t}$. We deduce from (2.5)(2.6) that there exists $c>0$ such that

$$
|\rho(x, v)| \geq c a(x)|v| \text { if }|v| \geq h(1),
$$

because

$$
\frac{|\rho(x, v)|}{|v|} \geq a(x) \text { if }|v| \geq 1
$$

and, if $h(1) \leq 1$,

$$
\frac{|\rho(x, v)|}{|v|} \geq a(x) \frac{g(|v|)}{|v|} \geq c a(x) \text { if }|v| \in[h(1), 1] .
$$

So we have

$$
\begin{aligned}
& \int_{S}^{T} E \phi^{\prime} \int_{\Omega_{1,3}^{t}} a u^{\prime 2} d x d t \leq \frac{1}{c} \int_{S}^{T} E \phi^{\prime} \int_{\Omega_{1,3}^{t}} u^{\prime} \rho\left(x, u^{\prime}\right) d x d t \\
& \leq \frac{\phi^{\prime}(S)}{c} \int_{S}^{T} E\left(-E^{\prime}\right) \leq C E(S)^{2} .
\end{aligned}
$$

Next we look at the part on $\Omega_{1,2}^{t}$ : by monotonicity, if $x \in \Omega_{1,2}^{t}$, then

$$
\phi^{\prime}(t)=g(h(t)) \leq\left|g\left(u^{\prime}\right)\right| \text {. }
$$

Since

$$
a(x) u^{\prime 2}\left|g\left(u^{\prime}\right)\right| \leq u^{\prime 2}\left|\rho\left(x, u^{\prime}\right)\right| \leq u^{\prime} \rho\left(x, u^{\prime}\right) \text { if }\left|u^{\prime}\right| \leq 1,
$$

and, if $h(1) \geq 1$,

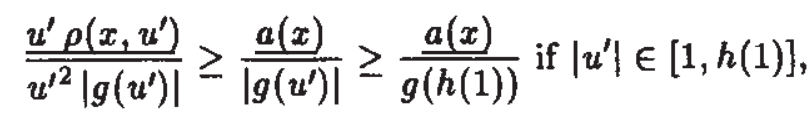

we have

$$
\begin{aligned}
& \int_{S}^{T} E \phi^{\prime} \int_{\Omega_{1,2}^{\mathrm{t}}} a u^{\prime 2} d x d t \leq \int_{S}^{T} E \int_{\Omega_{\mathrm{i}, 2}^{\mathrm{t}}} a\left|g\left(u^{\prime}\right)\right|{u^{\prime}}^{2} d x d t(6.11) \\
& \leq C \int_{S}^{T} E \int_{\Omega_{\mathrm{i}, 2}} u^{\prime} \rho\left(x, u^{\prime}\right) d x d t \leq C E(S)^{2}
\end{aligned}
$$


At last we look at the part on $\Omega_{1,1}^{t}$ :

$$
\begin{aligned}
& \int_{S}^{T} E \phi^{\prime} \int_{\Omega_{1,1}^{t}} a u^{\prime 2} d x d t \leq c \int_{S}^{T} E(t) \phi^{\prime}(t)\left(\int_{\Omega_{1,1}^{t}} h(t)^{2} d x\right) d t \\
& \leq c|\Omega| E(S) \int_{S}^{T} \phi^{\prime}(t)\left(g^{-1}\left(\phi^{\prime}(t)\right)\right)^{2} d t
\end{aligned}
$$

Since $a(x) \geq \alpha>0$ on $\omega$, we add $(6.10)-(6.12)$ to conclude.

Next we estimate the term

$$
\int_{S}^{T} E \phi^{\prime} \int_{\Omega} \rho\left(x, u^{\prime}\right)^{2} d x d t
$$

Lemma 12. There exists $C>0$ such that

$\int_{S}^{T} E \phi^{\prime} \int_{\Omega} \rho\left(x, u^{\prime}\right)^{2} d x d t \leq C E(S)^{2}+C E(S) \int_{S}^{T} \phi^{\prime}(t)\left(g^{-1}\left(\phi^{\prime}(t)\right)\right)^{2} d t$

Proof of Lemma 12. We follow the same idea: fix $t \geq 1$ and define

$$
\begin{gathered}
\Omega_{2,1}^{t}:=\left\{x \in \Omega:\left|u^{\prime}\right| \leq \phi^{\prime}(t)\right\}, \\
\Omega_{2,2}^{t}:=\left\{x \in \Omega: \phi^{\prime}(t)<\left|u^{\prime}\right| \leq \phi^{\prime}(1)\right\}, \\
\Omega_{2,3}^{t}:=\left\{x \in \Omega:\left|u^{\prime}\right|>\phi^{\prime}(1)\right\} .
\end{gathered}
$$

With this partition of $\Omega$ we easily see that

$$
\begin{aligned}
& \int_{S}^{T} E \phi^{\prime} \int_{\Omega_{2,3}^{t}} \rho\left(x, u^{\prime}\right)^{2} d x d t \leq C \int_{S}^{T} E \phi^{\prime} \int_{\Omega_{2,3}^{t}} u^{\prime} \rho\left(x, u^{\prime}\right) d x d t \\
& \leq C \int_{S}^{T} E\left(-E^{\prime}\right) \leq C E(S)^{2}
\end{aligned}
$$

Next we look at the part on $\Omega_{2,2}^{t}$ : by monotonicity, if $x \in \Omega_{2,2}^{t}$, then

$$
\begin{aligned}
& \int_{S}^{T} E \phi^{\prime} \int_{\Omega_{2,2}^{t}} \rho\left(x, u^{\prime}\right)^{2} d x d t \leq \int_{S}^{T} E \int_{\Omega_{2,2}^{t}}\left|u^{\prime}\right| \rho\left(x, u^{\prime}\right)^{2} d x d t \\
& \leq C \int_{S}^{T} E \int_{\Omega_{2,2}^{t}} u^{\prime} \rho\left(x, u^{\prime}\right) d x d t \leq C E(S)^{2}
\end{aligned}
$$


At last we look at the part on $\Omega_{2,1}^{t}$ :

$$
\begin{aligned}
& \int_{S}^{T} E \phi^{\prime} \int_{\Omega_{2,1}^{t}} \rho\left(x, u^{\prime}\right)^{2} d x d t \leq c \int_{S}^{T} E(t) \phi^{\prime}(t)\left(\int_{\Omega_{2,1}^{t}}\left(g^{-1}\left(\left|u^{\prime}\right|\right)\right)^{2} d x\right) d t \\
& \leq c|\Omega| E(S) \int_{S}^{T} \phi^{\prime}(t)\left(g^{-1}\left(\phi^{\prime}(t)\right)\right)^{2} d t .
\end{aligned}
$$

It is sufficient to add $(6.17),(6.18)$ and $(6.19)$ to conclude. (Note that if $\phi^{\prime}(1) \neq 1$, we repeat the arguments used to prove (6.10)(6.12).)

Assume that $\phi$ satisfies the following additional property:

$$
\int_{1}^{\infty} \phi^{\prime}(t)\left(g^{-1}\left(\phi^{\prime}(t)\right)\right)^{2} d t \text { converges. }
$$

This property is closely related to the growth of $g$ near $\mathbf{0}$ and the decay rate of $\phi^{\prime}$ at infinity. Then we deduce from (5.3) and the estimates (6.5)-(6.13) that there exists a positive constant $C$ such that

$$
\forall 1 \leq S<T, \int_{S}^{T} E(t)^{2} \phi^{\prime}(t) d t \leq C E(S) .
$$

This gives a first estimate on the decay rate of the energy: we can apply the Gronwall type inequality (4.2) with $\sigma=1$ and we deduce that there exists $C$ depending on $E(0)$ such that

$$
\forall t \geq 1, E(t) \leq \frac{C}{\phi(t)}
$$

So the problem is to find a strictly increasing function $\phi$ satisfying the following conditions

$$
\begin{gathered}
\phi \text { is concave and } \phi(t) \longrightarrow+\infty \text { as } t \longrightarrow+\infty \\
\phi^{\prime}(t) \longrightarrow 0 \text { as } t \longrightarrow+\infty \\
\int_{1}^{+\infty} \phi^{\prime}(t)\left(g^{-1}\left(\phi^{\prime}(t)\right)\right)^{2} d t \text { converges }
\end{gathered}
$$


and then to estimate the growth of $\phi$ at infinity in order to prove (2.15). If such a function exists, we can assume that $\phi(1)=1$. With the change of variable defined by

$$
\tau=\phi(t)
$$

we see that

$$
\begin{aligned}
& \int_{1}^{+\infty} \phi^{\prime}(t)\left(g^{-1}\left(\phi^{\prime}(t)\right)\right)^{2} d t=\int_{1}^{+\infty}\left(g^{-1}\left(\phi^{\prime}\left(\phi^{-1}(\tau)\right)\right)\right)^{2} d \tau \\
& =\int_{1}^{+\infty}\left(g^{-1}\left(\frac{1}{\left(\phi^{-1}\right)^{\prime}(\tau)}\right)\right)^{2} d \tau
\end{aligned}
$$

Let us define $\psi$ by

$$
\forall t \geq 1, \psi(t)=1+\int_{1}^{t} \frac{1}{g\left(\frac{1}{\tau}\right)} d \tau
$$

Then $\psi$ is a strictly increasing function of class $\mathcal{C}^{2}$ that satisfies

$$
\psi^{\prime}(t)=\frac{1}{g\left(\frac{1}{t}\right)} \longrightarrow+\infty \text { as } t \rightarrow+\infty
$$

So

$$
\psi(t) \longrightarrow+\infty \text { as } t \longrightarrow+\infty
$$

and

$$
\int_{1}^{+\infty}\left(g^{-1}\left(\frac{1}{\psi^{\prime}(\tau)}\right)\right)^{2} d \tau=\int_{1}^{+\infty} \frac{1}{\tau^{2}} d \tau<+\infty
$$

Moreover $\psi^{\prime}$ is increasing so $\psi$ is a convex function. Then it is easy to verify that $\psi^{-1}$ is concave: derivating two times the expression

$$
\psi\left(\psi^{-1}(\tau)\right)=\tau
$$

we see that

$$
\left(\psi^{-1}\right)^{\prime \prime}(\tau)=-\frac{\psi^{\prime \prime}\left(\psi^{-1}(\tau)\right)\left(\left(\psi^{-1}\right)^{\prime}(\tau)\right)^{2}}{\psi^{\prime}\left(\psi^{-1}(\tau)\right)}=-\frac{\psi^{\prime \prime}\left(\psi^{-1}(\tau)\right)}{\left(\psi^{\prime}\left(\psi^{-1}(\tau)\right)\right)^{3}} \leq 0 .
$$


That is why we define

$$
\forall t \geq 1, \phi(t)=\psi^{-1}(t)
$$

Then $\phi$ is a strictly increasing concave function of class $\mathcal{C}^{2}$ that satisfies all the assumptions we made in our computations: (6.23) and (6.25) are already verified, $(6.24)$ is also true because

$$
\phi^{\prime}(t)=\frac{1}{\psi^{\prime}(\overline{\phi(t)})}=g\left(\frac{1}{\phi(t)}\right) \longrightarrow 0 \text { when } t \longrightarrow+\infty .
$$

Note that $\phi(1)=1$ because $\psi(1)=1$. So we have explicitly constructed a function $\phi$ that satisfies the required properties. With that special choice we have

$$
\begin{aligned}
& \int_{S}^{T} E(t)^{2} \phi^{\prime}(t) d t \leq C E(S)^{2}+C E(S) \int_{S}^{+\infty}\left(g^{-1}\left(\frac{1}{\left(\phi^{-1}\right)^{\prime}(\tau)}\right)\right)^{2} d \tau \\
& \leq C E(S)^{2}+C E(S) \int_{\phi(S)}^{+\infty} \frac{1}{\tau^{2}} d \tau=C E(S)^{2}+C \frac{E(S)}{\phi(S)}
\end{aligned}
$$

Then we can apply Lemma 3 with $\sigma=\sigma^{\prime}=1$ to deduce that

$$
E(t) \leq \frac{C}{\phi(t)^{2}}
$$

It remains to estimate the growth of $\phi$. This is equivalent to majorate the function $\phi^{-1}=\psi$. Set $\tau_{0}$ such that

$$
g\left(\frac{1}{\tau_{0}}\right) \leq 1
$$

By monotonicity we have

$$
\forall \tau \geq \tau_{0}, \psi(\tau) \leq 1+(\tau-1) \frac{1}{g\left(\frac{1}{\tau}\right)} \leq \tau \frac{1}{g\left(\frac{1}{\tau}\right)}=\frac{1}{G\left(\frac{1}{\tau}\right)}
$$

So

$$
\frac{1}{\phi(t)} \leq \frac{1}{\tau}
$$

with

$$
t=\frac{1}{G\left(\frac{1}{\tau}\right)} \text { so } \frac{1}{\tau}=G^{-1}\left(\frac{1}{t}\right) \text {. }
$$

Thus the proof of (2.15) is achieved. 


\subsection{Improved estimate on the decay rate of the energy}

When $H$ is an increasing function whose value in zero is zero, let us now define

$$
h(t)=H^{-1}\left(\phi^{\prime}(t)\right)
$$

Since we have

$$
\phi^{\prime}(t) u^{\prime 2} \leq H\left(u^{\prime}\right) u^{\prime 2}=u^{\prime} g\left(u^{\prime}\right)
$$

on $\Omega_{1,2}^{t}$, the same reasoning holds. So with

$$
\phi^{-1}(t)=1+\int_{1}^{t} \frac{1}{H\left(\frac{1}{\tau}\right)} d \tau
$$

we see that

$$
E(t) \leq C\left(g^{-1}\left(\frac{1}{t}\right)\right)^{2},
$$

which gives (2.16) that is a better estimate than (2.15).

So the proof of Theorem 1 is achieved.

\subsection{The case of the polynomial growth}

If $g(x)=x^{p}$ for some $p>1$ on $(0,1)$, then $H(x)=x^{p-1}$ is increasing. So (2.16) gives the estimate

$$
E(t) \leq \frac{C}{t^{2 / p}}
$$

We did not manage to find a function $\phi$ that gives directly the estimate first found by M. Nakao [19] or A. Haraux [7] in the case of internal damping or L.R. Tcheugoué Tébou [21] for localized damping:

$$
E(t) \leq \frac{C}{t^{2 /(p-1)}}
$$

However the method leading to (2.16) allows us to find again a decay rate as close as we want than this one: set

$$
n \geq 1,
$$


and define

$$
\phi_{n}(t)=t^{1 /(1+n(p-1))} \text { if } t \geq 1 .
$$

$\phi_{n}$ is chosen so that

$$
H^{-1}\left(\frac{\frac{1}{\left(\phi_{n}^{-1} \cdot\right)^{\prime}(r)}}{r^{n}}=\frac{1}{r^{n}}\right.
$$

Moreover we see that $\phi_{n}$ is a concave function that satisfies (6.4). So we derive from (6.1) that $E$ satisfies

$$
\begin{aligned}
& \int_{S}^{T} E(t)^{2} \phi_{n}^{\prime}(t) d t \leq c E(S)^{2}+C E(S) \int_{S}^{T} \phi_{n}^{\prime}(t)\left(H^{-1}\left(\phi_{n}^{\prime}(t)\right)\right)^{2} d t \\
& \leq c E(S)^{2}+C E(S) \int_{\phi_{n}(S)}^{\phi_{n}(T)} \frac{1}{\tau^{2 n}} d \tau \leq c E(S)^{2}+2 C n \frac{E(S)}{\phi_{n}(S)^{2 n-1}} .
\end{aligned}
$$

Then we apply Lemma 3 with $\sigma=1$ and $\sigma^{\prime}=2 n-1$ to deduce that

$$
E(t) \leq \frac{C_{n}}{\phi_{n}(t)^{2 n}}=C_{n} t^{\frac{-2 n}{n(p-1)+1}}
$$

It is sufficient to choose $n$ large enough to obtain a decay rate close enough than the classical one.

\section{References}

[1] M. Aassila, A new approach of strong stabilization of distributed systems, Differential and Integral Equations, 11 (1998), 369-376.

[2] M. Aassila, Decay estimates for the wave equation with a nonlinear nonmonotone weak damping, Applicable Analysis, to appear.

[3] C. Bardos, G. Lebeau, J. Rauch (1988), Un exemple d'utilisation des notions de propagation pour le contrôle et la stabilisation des problèmes hyperboliques, the procedings of the workshop on non linear hyperbolic equations in applied scinces (edited by N. Bellono), Rendiconti del Seminario Matematico, Universita'e Politecnico Torino (Fascicolo Speciale 1988), 11-31. 
[4] F. Conrad et B. Rao (1993), Decay of solutions of wave equations in a star-shaped domain with non-linear boundary feedback, Asymptotic Analysis. 7 (1993), 159-177.

[5] C.M. Dafermos (1978), Asymptotic behavior of solutions of evolutions equations, Nonlinear evolution equations (M.G. Crandall ed.), Academic Press, New-York, p. 103-123.

[6] P. Grisvard, Elliptic problems in nonsmooth domains, Monographs and studies in Math., 24, Pitman, Boston, 1985.

[7] A. Haraux (1978), Comportement à l'infini pour une équation des ondes non linéaire dissipative. C. R. Acad. Sci. Paris, 287, série A, p. 507-509.

[8] A. Haraux (1978), Oscillations forcées pour certains systèmes dissipatifs non linéaires, Publication du Laboratoire d'Analyse Numérique No. 78010, Université Pierre et Marie Curie, Paris.

[9] A. Haraux, (1980), Nonlinear evolution equations, Lecture Notes in Mathematics 841, Springer-Verlag Berlin Heidelberg New-York.

[10] A. Haraux, (1985), Stabilization of trajectories for some weakly damped hyperbolic equations J. of Diff. Eq., 59,

[11] V. Komornik (1994), Exact Controllability and Stabilization. The Multiplier Method, John Wiley, Chicester and Masson, Paris.

[12] I. Lasiecka and D. Tataru (1993), Uniform boundary stabilization of semilinear wave equation with nonlinear boundary damping, Lectures Notes in Pure and Applied Maths 142, Dekker, New York.

[13] J. L. Lions (1988), Contrôlabilité exacte et stabilisation de systèmes distribués, Vol.1, Masson, Paris.

[14] K. Liu (1997), Locally distributed control and damping for the conservative systems, SIAM J. Control Opt. 35, No.5, 1997.

[15] K. Liu and M. Yamamoto (1997), On observation time duration for exact internal observability by the piecewise multiplier method, preprint of the Universit of Tokyo, 1997. 
[16] P. Martinez, submitted.

[17] M. Nakao (1995), Energy decay for the wave equation with a nonlinear weak dissipation, Differential and Integral Equations, Vol. 8, No. 3, p. 681-688.

[18] M. Nakao (1996), Decay of solutions of the wave equation with a local degenerate dissipation, Israel J. of Maths 95, 1996.

[19] M. Nakao (1996), Decay of solutions of the wave equation with a local nonlinear dissipation, Math. Ann. 305, No.3, 1996, 403-417.

[20] A. Pazy, Semigroups of linear operators and applications to partial differential equations, Springer, New-York, 1983.

[21] L.R. Tcheugoué Tébou (1997), Estimations d'énergie pour l'équation des ondes avec un amortisseent non linéaire localisé, C. R. Acad. Sci. Paris, t. 325, Série 1, p. 1175-1179.

[22] E. Zuazua, Chap. 7 in Lions (1988).

[23] E. Zuazua (1990), Exponential decay for the semi-linear waveequation with locally distributed damping, Comm. P.D.E. 15 (1990), 205-235.

Institut de Recherche Mathématique Avancée

Université Louis Pasteur, 7

rue René Descartes

67084 Strasbourg Cédex

France

$e$-mail: martinezemath.u-strasbg.fr

Recibido: 15 de Abril de 1998

Revisado: 27 de Mayo de 1998 